\title{
SYSTEMATIC DIFFERENCES INSTRUMENT MINUS FK5 IN THE SOUTHERN HEMISPHERE
}

\author{
G. CARRASCO AND P. LOYOLA \\ Observatorio Astronómico Nacional, Cerro Calán \\ Departamento de Astronomia, Universidad de Chile \\ Casilla 36-D \\ Santiago \\ Chile
}

\section{Introduction}

Observations of Fundamental Faint Star Catalogue (FKSZ) stars, made with the Repsold Meridian Circle at Cerro Calán National Astronomical Observatory, began in 1979 and finished in 1988. Today International Reference Star (IRS) observations are in progress. These observations correspond to the second epoch of the Santiago 67 Catalogue (Carrasco and Loyola 1981) and they are going to be used for determining the proper motions of these stars.

During this period, a series of fundamental FK5 stars along the whole meridian arc were periodically observed, in order to determine the instrumental system. The stars were observed differentially and the reductions were made according to the Zverev quasi-absolute method (1965, 1969).

The results of the observations in right ascension, of the series of fundamental stars, are given. The systematic differences $\Delta \alpha \cos \delta$, in the sense Instrument $-F K 4$ and Instrument $-F K 5$, are presented. The mean epoch of the observations is 1982.2.

\section{Observations and Reductions}

From January 1979 to June 1989 , three observers carried out observations of a series of 237 FK5 stars, with a total number of 9768 individual observations, in the zone $+41^{\circ}>\delta>-90^{\circ}$ in upper culmination and $-90^{\circ}<\delta<-68^{\circ}$ in lower culmination.

In order to investigate the behavior of the parameter $n$ of the Bessel formula, a preliminary reduction of the observations was made. These values of $n$ were corrected for the rate with declination and a second approximation was calculated (Anguita et al. 1975).

The instrumental coordinate system was obtained using the quasi-absolute method, which imposes the following conditions: that the instrumental system must be forced to coincide with the fundamental system at the equator and at the pole. An instrumental system was obtained in the FK4 and FK5 systems and the differences $\Delta \alpha \cos \delta$ for each observed star was computed.

The differences $\Delta \alpha \cos \delta$ obtained for each star were grouped in $5^{\circ}$ declination zones. All residuals larger than $3 \sigma\left(\sigma_{\alpha}=0.015 \cos \delta\right)$ were eliminated after a careful investigation of the observational 
data. The mean values for each zone, for the two positions of the instrument and in the FK4 and FK5 systems are plotted in Figure 1. For these differences, the mean standard deviations are 0.022 for the FK4 and 0.017 for the FK5. No clamp corrections were applied.

From Figure 1 it is possible to see that, in general, the FK5 system shows a better agreement than the FK4 system with the Repsold Meridian Circle system, especially in the zone $-40^{\circ}$ to $-70^{\circ}$. This agreement is broken in the zone $-70^{\circ}$ to $-85^{\circ}$.

Acknowledgements. This project was supported by the Fondo Nacional de Desarrollo Cientifico y Tecnológico, FONDECYT (Projects No. 337/87, 490/88 and 976/89) and Universidad de Chile, Departamento Técnico de Investigación (DTI Project E-1935).

\section{References}

Anguita, C., Carrasco, G., Loyola, P., Bedin, V.N., Naumova, A.A., Polojentsev, D.D., Polojentseva, T.A., Tavastsherna, K.N., and Zverev, M.S.: 1975, Publ. Dep. Astron. Univ. Chile 2, 181. Carrasco, G., Loyola, P.: 1981, Publ. Dep. Astron. Univ. Chile 4.

Zverev, M.S.: 1965, Astron. Zh. 42, 823.

Zverev, M.S.: 1969, Astron. Zh. 46, 129 (A.J. 13, 1008).

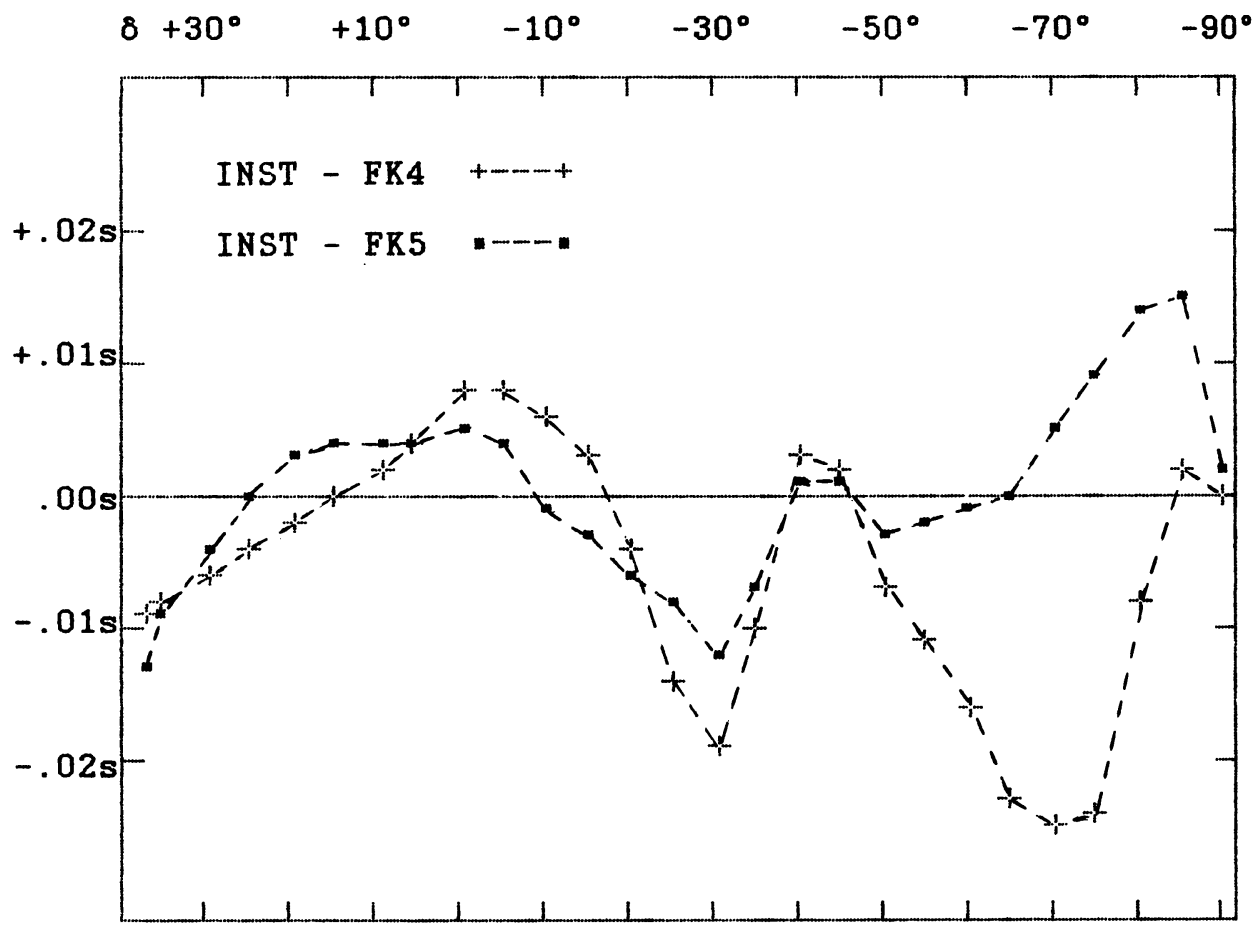

Figure 1. Systematic differences $\Delta \alpha \cos \delta$ in the sense Instrument-FK4 and Instrument-FK5. 